\title{
Computer science and technology literature companion to Swartz, MIT and JSTOR.
}

Camille Akmut

January 19, 2020

$\frac{\underline{\text { Abstract }}}{\text { Commentary, edition of relevant textbooks (DHCP/IP, } \lambda, \text { etc.). }}$

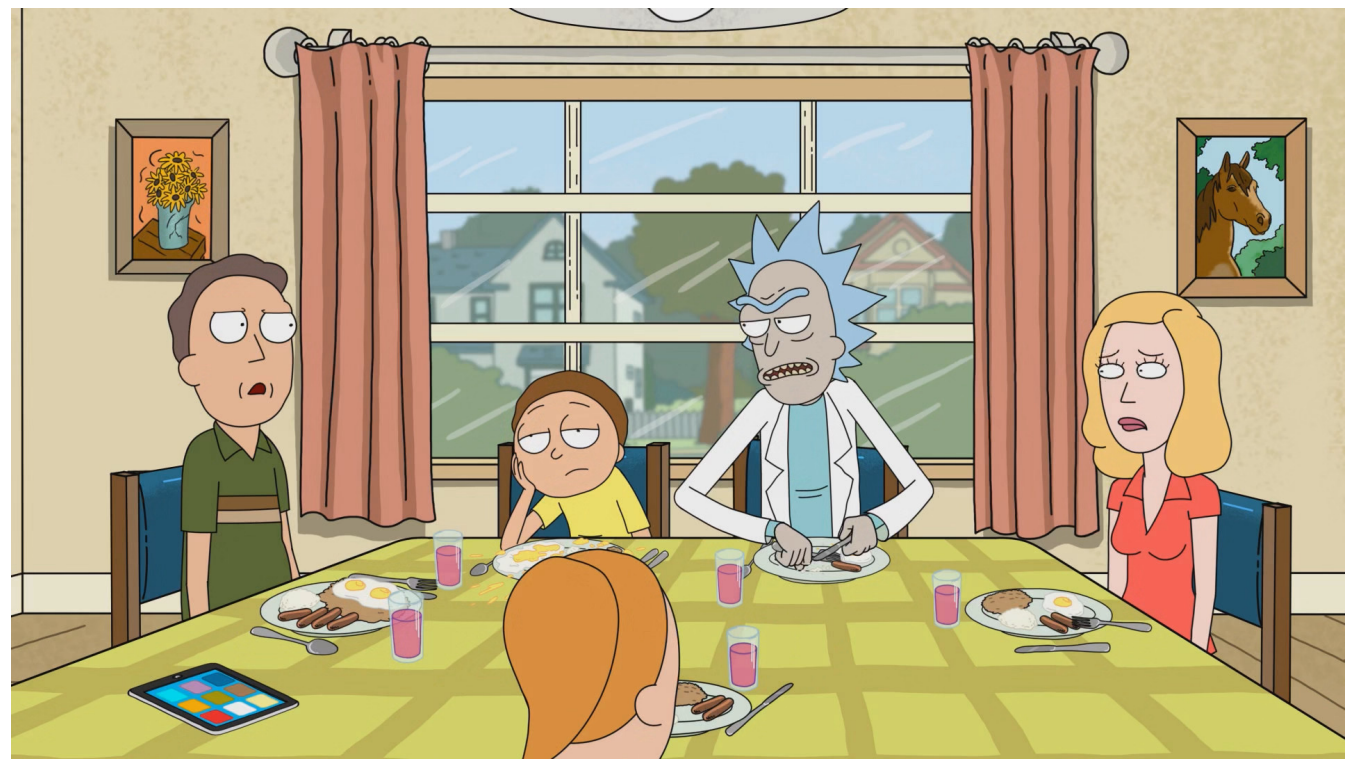

"I'll tell you how I feel about school, Jerry. ...

I mean, it's, it's, it's not a place for smart people ..." 


\section{Int. : On schools, books, and a queer scientist}

Aaron Swartz dropped out of Stanford after only one year -

He gave "Sociology" as his subject ${ }^{1}$.

He called that institution the academic equivalent of a "Disneyland", and described in painful minutiae the details of the private education he had - to his own dismay - known, and discovered there. ${ }^{23}$

He liked libraries, where he found books that changed his life ${ }^{4}$.

He did not distinguish between "high" and "low" cultures, and liked both. ${ }^{5}$

In that he was representative of contemporary computer scientists and technologists - of whom we have shown in abundance just how important popular culture is to them; both increasingly starting to reflect one another.

He also echoed feelings expressed by Lawrence Lessig ${ }^{6}$, who we cannot rightfully call a mentor, as both seemed to learn much from each other - despite the fact that one was just a teenager and the other a professor.

Would he have liked (the first episode) of Rick and Morty? And, what kind of essays would he write today? We won't know.

He was twenty years old when the paper avalanche of the Justice system started progressively covering him. He died a few years later;

And, only MIT professors could earnestly wonder why he did it, while trying to disculpate their own institution, in a neutral Report ${ }^{7}$.

E. M. Forster had this to say about certain scholarly institutions (Cambridge, in his case) which have the potential to very quickly turn into golden prisons :

"I don't think it's a place in which [one] ought to remain in. (...) [One] ought to go out into the world. (...) It's most necessary for the writer, and for everyone else." 8

Your author knew too. And, this was the best thing they did by far. They experienced a love like Divine Comedy's Beatrice, and then discovered a new science.

\footnotetext{
${ }^{1}$ See : CV in the FBI File.

${ }^{2}$ Cf. multi-part blog series on his first week(s) at "Stanford".

${ }^{3}$ (Personal note : If Bambi was the best Disney was able to produce, then Disneyland cannot amount to much... (And, neither can Stanford, by derivation.))

${ }^{4}$ See blog entry "The Book That Changed My Life".

${ }^{5}$ His love of Buffy (old blog).

${ }^{6}$ Lessig on e.g. manga in his book Free culture.

${ }^{7} \mathrm{Hal}$ Abelson at al., 2013, Report to the president. (The naive questions about "why" are in part 2, I believe.)

${ }^{8}$ Interview with the BBC (1958).
} 
In networking terminology, the host describes here the user i.e. Aaron Swartz's computer (2 laptops*). The other, opposite term is server i.e. MIT's computers.

[*an Acer brand laptop, "ghost laptop", and a Macbook, "ghost macbook" (see a.o. the Government's consolidated response, and the closet photos...).]

B. WARD, HOW LINUX WORKS p. 214

When you set a network host to get its configuration automatically from the network, you're telling it to use the Dynamic Host Configuration Protocol (DHCP) to get an IP address, subnet mask, default gateway, and DNS servers. Aside from not having to enter these parameters by hand, DHCP has other advantages for a network administrator, such as preventing IP address clashes and minimizing the impact of network changes. It's very rare to see a modern network that doesn't use DHCP.

For a host to get its configuration with DHCP, it must be able to send messages to a DHCP server on its connected network. Therefore, each physical network should have its own DHCP server ...

Note When making an initial DHCP request, a host doesn't even know the address of a DHCP server, so it broadcasts the request to all hosts (usually all hosts on its physical network).

When a machine asks a DHCP server for an IP address, it's really asking for a lease on an address for a certain amount of time.

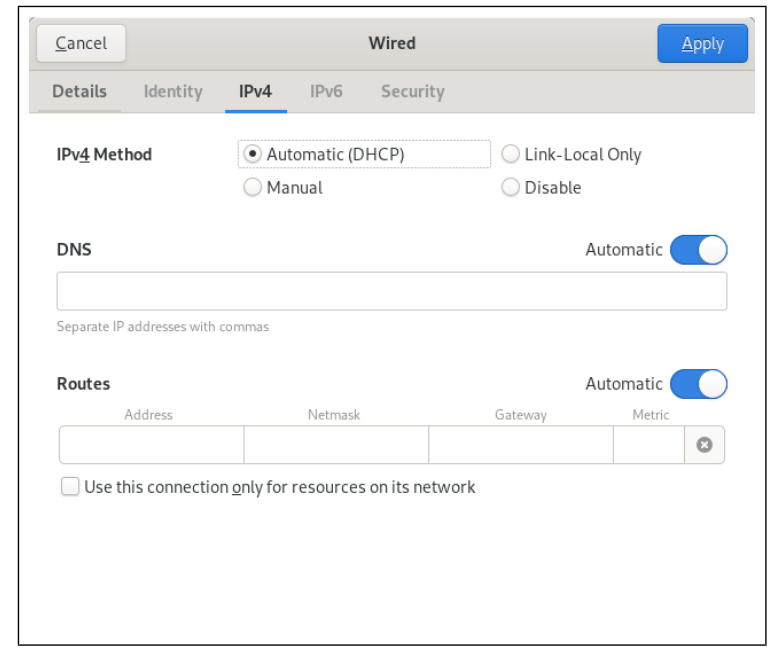

Figure 1: The relevant sections in Debian GNU/Linux (host perspective) 
Alternatively the terminology "client" is also used (to describe here still Aaron's computer(s)).

LPI LINUX CERTIFICATION IN A NUTSHELL pp. 390-391

The Dynamic Host Configuration Protocol (DHCP) is a protocol extension of the BOOTP protocol, which provides automated IP address assignment (among other things) to client systems on a network. It handles IP address allocation in one of three ways:

Dynamic allocation

In this scheme, a DHCP server maintains a preset list of IP addresses designated by the system administrator.

Manual allocation

Automatic allocation

This method assigns a permanent address to a client.

Here's an overview of how it works:

1. A DHCP client sends a broadcast message to the network to discover a DHCP server.

2. One or more DHCP servers respond to the request via their own broadcast messages, offering an IP address to the client.

3 . The client chooses one of the servers and broadcasts an acknowledgment, requesting the chosen server's identity.

4. The selected server logs the connection with the client

DHCPOFFER on 18.2.55.212 to 00:23:5a:73:5f:fc (ghost-laptop) via 18.55.0.1

DHCPOFFER on 18.2.55.166 to 00:17:f2:2c:b0:74 (ghost-macbook) via 18.55.0.1

Figure 2: USA v. Swartz exhibit (1)

when a client receives a dynamically assigned IP address from a DHCP server, the address is said to be leased for a finite duration. The length of a DHCP lease is configurable by the system administrator and typically lasts for one or more days.

When a lease expires without being renegotiated by the client, it as assumed that the client system is unavailable, and the address is put back into the free pool of addresses. A lease may also be terminated by a client that no longer needs the IP address, in which case it is released.

The DHCP server process is called dhcpd. It is typically started at boot time and listens for incoming DHCP request broadcasts. 
keepgrabbing.py was written around a lambda function, its core.

J. GUTTAG, INTRODUCTION TO COMPUTATION... ch. 4, ch. 5

The name "lambda abstraction" is derived from mathematics developed by Alonzo Church in the 1930s and 1940s.

The general form of a lambda expression is

lambda <sequence of variable names>: <expression>

For example, the lambda expression lambda $\mathrm{x}, \mathrm{y}: \mathrm{x} * \mathrm{y}$ returns a function that returns the product of its two arguments. Lambda expressions are frequently used as arguments to higher-order functions.

For example, the code

$\mathrm{L}=[]$

for $i$ in map(lambda $x, y: x * * y,[1,2,3,4],[3,2,1,0]$ ): L.append(i) print (L)

prints $[1,4,3,1]$.

UNIX AND LINUX SYSTEM ADMINISTRATION pp. 402-409

When setting up a new DHCP server, you must also make sure that an empty lease database file has been created. (...) It's usually somewhere underneath / var.

'Gary Host','ghost42@mailinator.com',",','NULL,NULL,10,'13-Oct-2010',",'02-Oct-2010','10:20:37',

l,'Grace Host','ghost42@mailinator.com',",",NULL,NULL,5,'13-Oct-2010',",'08-Oct-2010','22:13:26'

Figure 3: USA v. Swartz exhibit (2)

Security issues

IP spoofing

... $^{9}$

${ }^{9}>$ "is usually associated with some kind of playful, clever network behavior" - we would have written, instead. 


\section{REFERENCES}

- Guttag, John. 2016. Introduction to Computation and Programming Using Python. MIT Press.

- Haeder et al.. 2010. LPI Linux Certification in a nutshell. O'Reilly.

- Nemeth et al.. 2018. UNIX and Linux System Administration Handbook.

Pearson.

- Ward, Brian. 2015. How Linux Works. No Starch Press. 